\title{
Prevalence and Antifungal Susceptibility patterns of Candida isolated on CHROMagarTMCandida at a tertiary referral hospital, Eastern Uganda.
}

Juliet Watsemwa

Makerere University

Jacob Iramiot

Busitema University

JOHN BOSCO KALULE ( $\square$ kaluleb@gmail.com )

Makerere University https://orcid.org/0000-0003-3409-4327

Research article

Keywords:

Posted Date: May 6th, 2019

DOl: https://doi.org/10.21203/rs.2.9473/v1

License: (c) (i) This work is licensed under a Creative Commons Attribution 4.0 International License.

Read Full License 


\section{Abstract}

Background Pregnant women are susceptible to vaginal candidiasis and maternal vulvovaginal candidiasis is a major risk factor for colonization and/or infection of the infant. The purpose of this study was to determine the prevalence and antifungal patterns of albicans and non-albicans Candida among pregnant women attending a tertiary referral hospital. Methods Vaginal discharge- cotton swabs were self-collected from pregnant women clinically proven to have vulvovaginal candidiasis at the antenatal clinic of a tertiary referral hospital between January and July 2018. Microscopy and culture on Sabouraud's Dextrose Agar with chloramphenicol was done on the vaginal discharge-cotton swabs. Confirmatory fungal identification was done using CHROM agarTM Candida. Antifungal susceptibility testing was carried out using the standardized Kirby Bauer dilution method. Results Candida were isolated from $50.81 \%(126 / 249)$ of the swabs and included C. albicans $(80.16 \%, 101 / 126)$, C. glabrata (19.05\% (24/126) and C. krusei $(0.79 \%, 1 / 126)$. Of the women from whom Candida were isolated, $11.1 \%$ $(14 / 126)$ were in the first trimester, $39.7 \%$ (50/126) were in the second, while $49.2 \%(62 / 126)$ were in the third. Of the Candida isolates, $80.16 \%$ (101/126) were C. albicans, $19.05 \%(24 / 126)$ were C. glabrata and $0.79 \%(1 / 126)$ were C. krusei. Overall, all the isolates were non-susceptible to Amphotericin B, while $60.3 \%$ (76/126), 50\% (63/126), 62.7\% (79/126), and 48.4\% (61/126) were non-susceptible to itraconazole, fluconazole, nystatin, and clotrimazole respectively. All the non-albicans Candida were resistant to itraconazole, amphotericin $\mathrm{B}$, and fluconazole. Conclusion Vulvovaginal candidiasis due to multidrug resistant C.glabrata among pregnant women will require that treatment regimes are adjusted to carter for the recurrent forms. The use of CHROMagarTMCandida media for identification of clinically relevant Candida should be adopted instead of conventional methods that are tedious and time consuming such that treatment is based on laboratory evidence.

\section{Background}

Maternal vulvovaginal candidiasis is a major risk factor for Candida colonization and infection of the infant and has been linked to perinatal morbidity and mortality in the infants.[1] Recent sexual intercourse and use of injectable contraceptives are risk factors for colonization in pregnant women.[2] Congenital transmission of Candida from the infected mother's vagina to the newborn can occur. Therefore, pregnant women, especially those that are multigravida and diabetic, ought to be screened for vulvovaginal candidiasis (VVC) irrespective of symptomatic status.[3] Approximately $28.4 \%$ of all pregnant women in the third trimester get colonized by Candida and in $89.7 \%$ (44 women) of the cases, the colonization is due to an overgrowth of Candida albicans.[4]

C. albicans is the most common and clinically relevant pathogen that is responsible for $85-90 \%$ of the VVC cases. $[5,6]$ The formation of a germ tube is necessary for successful colonization of the vaginal mucosa.[7] Moreover, this phenotype switching phenomenon is associated with alterations in antifungal susceptibility patterns.[8] And yet pregnant women have a limited spectrum of drugs that they can use especially in the later stages of pregnancy.[9] 
More recently, due to resistance to commonly used antifungals, non- albicans Candida have emerged as clinically relevant causes of Candidiasis.[10] Most prevalent among these is $C$. glabrata [5, 6, 11-13] which is highly resistant to the commonly used azole antifungals.[10] $C$. krusei infections are commonest among patients with hematologic malignancies [14] and those of advanced age.[15] Additionally, VVC due to non-albicans Candida is often recurrent drug to their antifungal resistance to the drugs but could be managed using boric acid [16] or antifungal plant extracts [17] or prevented through the use of vaginal probiotics.[18] In Kenya, the frequency of $C$. glabrata (29.79\%) in VVC was much higher than that for $C$. krusei (2.13\%), C. tropicalis (3.19\%), and C. parapsilosis (1.06\%) [19] - findings which seem to contradict an earlier finding that $C$. tropicalis is one of the most common Candida species that causes in human disease, especially in tropical climates and its frequency varies per geographic region.[20]

Due to a thin laboratory infrastructure, there is scanty data on prevalence and antifungal patterns of Candida in Uganda. [21] Infections due to Candida are often treated empirically because the conventional identification methods for yeasts are tedious and long.[22] Out of concerns for colonization of the infants at birth, and the health of pregnant women in this region, this study piloted the use of a chromogenic media for identification of Candida to species level and determined the antifungal patterns of Candida isolated on CHROMagarTMCandida.

\section{Materials And Methods}

\section{Study setting}

This was a cross sectional study carried out among pregnant women attending the antenatal clinic at the Mbale Regional Hospital (MRRH), Uganda between January and July 2018. A total of 249 pregnant women were non randomly recruited by convenient sampling. The study relevance and procedure was explained to the participants in their local languages by a study nurse. Written consent was then sought from the study participants. For those below the age of 18 parental/ guardian guidance or consent was obtained before recruitment. Only consenting pregnant women with confirmed vulvovaginal candidiasis were enrolled into the study.

\section{Ethical approval}

Ethical approval of the study was received from the MRRH research and ethics Committee (MRRH/12/2018) and the research and ethics committee of the School of Biotechnical and Biomedical Laboratory Sciences, Makerere University (SBBLS/JBK/2018).

\section{Sample collection and transportation}

Self-collected vaginal discharge -cotton swabs from pregnant women were transported in sterile tubes in temperature -monitored boxes to the clinical microbiology laboratory and processed within 3 hours of collection. 


\section{Laboratory testing}

\section{Microscopy}

Microscopy on the vaginal discharge-cotton swabs to observe suspect yeast cells was carried out by two laboratory technologists based on the National System for External Quality Assessment (NSEQA) and the College of American Pathologists (CAP).[23]

Fungal culture, identification, and susceptibility testing

The swabs were streaked on SDA with chloramphenicol (HiMedia laboratories Pvt Ltd. India) and cultured at $37^{\circ} \mathrm{C}$ for $48 \mathrm{~h}$ and the resultant colonies gram stained to observe ovoid yeast cells and pseudohyphae. These were then regarded suspect Candida. A single colony was identified per patient.

Fungal identification was done using CHROM agarTM Candida (CHROM agar Company, France). Only one isolate was identified from per patient. Antifungal susceptibility to anti-fungal agents fluconazole $(25 \mu \mathrm{g})$, Itraconazole $(10 \mu \mathrm{g})$, clotrimazole $(10 \mu \mathrm{g})$, nystatin $(100 \mathrm{U})$, amphotericin B (100U) (Bioanalyze, Yenimahalle, Turkey) was performed using the kirby Bauer disc diffusion method and using 0.5 McFarland standard equivalent of inoculum. Mueller- Hinton agar with glucose (2\%) and methylene blue (5 mg L-1) was used and was supplemented with chloramphenicol (250 mg L-1). Innoculum suspensions were incubated at $37^{\circ} \mathrm{C}$ for 24 hours. The diameters of zones of inhibition were measured in millimeters using a ruler.[24] The results were interpreted according to Clinical Laboratory Standard Institute (CLSI) M44A document.[25] Commercially available control strains were used for each of the Candida species i.e C.krusei ATCC 6258, C.albicans ATCC 90028, C.glabrata ATCC 90030.

\section{Results}

\section{Sociodemographic characteristics of the study participants}

The average age of the participants in this study was $26.9 \pm 2.3 \mathrm{yrs}$.

Of the 249 pregnant women that consented to participate in the study, 9.2\% (23/249) were in the fist trimester, $41.4 \%$ (103/249) were in the second, while $48.9 \%$ (123/249) were in the third. Of these, $20.8 \%$ $(52 / 249)$ had used antibiotics in the past two weeks.

\section{Prevalence and phenotypic characterization of Candida}

Candida were isolated from $50.81 \%(126 / 249)$ of the swabs and included C. albicans $(80.16 \%, 101 / 126)$, C. glabrata $(19.05 \%(24 / 126)$ and C. krusei $(0.79 \%, 1 / 126)$. Of the 126 vaginal-discharge cotton swabs from as many women, from which Candida were isolated, $11.1 \%(14 / 126)$ were in the first trimester, $39.7 \%$ (50/126) were in the second, while 49.2\% (62/126) were in the third. Of the Candida isolates, $80.16 \%$ (101/126) were C. albicans, $19.05 \%$ (24/126) were C. glabrata and $0.79 \%(1 / 126)$ were C. krusei.

\section{Antifungal susceptibility patterns of isolated Candida species}


Overall, all the isolates were non-susceptible to Amphotericin B, while 60.3\% (76/126), 50\% (63/126), $62.7 \%$ (79/126), and $48.4 \%$ (61/126) were non-susceptible to itraconazole, fluconazole, nystatin, and clotrimazole respectively. All the non-albicans Candida were resistant to itraconazole, amphotericin B, and fluconazole.

Candida albicans showed non-susceptibility to Itraconazole (50.5\%, 51/101), amphotericin B (100\%, $101 / 101)$, fluconazole $(37.6 \%, 38 / 101)$, nystatin $(57.4 \%, 58 / 101)$, and clotrimazole $(39.6 \%, 40 / 101)$. Among the non-albicans Candida species, C. glabrata showed non-susceptibility to itraconazole (100\%, $24 / 24)$, amphotericin B $(100 \%, 24 / 24)$, fluconazole $(100 \%, 24 / 24)$, nystatin $(83.3 \%, 20 / 24)$, and clotrimazole $(83.3 \%, 20 / 24)$. The $C$. krusei isolate showed resistance to itraconazole, amphotericin $\mathrm{B}$, and fluconazole.

Table 1 Antifungal Susceptibility Patterns of Candida isolated from vaginal discharge -cotton swabs from pregnant women

\begin{tabular}{|c|c|c|c|c|}
\hline \multirow[t]{2}{*}{ Antifungal drug } & \multicolumn{3}{|c|}{ Species of Candida, n (\%) } & \multirow[b]{2}{*}{$\begin{array}{l}\text { Total }(\%, 95 \% \mathrm{Cl}) \\
\mathrm{N}=126\end{array}$} \\
\hline & C. albicans $(n=101)$ & C. glabrata $(\mathrm{n}=24)$ & C. krusei $(\mathrm{n}=1)$ & \\
\hline \multicolumn{5}{|l|}{ Itraconazole } \\
\hline Susceptible & $50(49.5)$ & 0 & 0 & $50(39.7,31.6-48.4)$ \\
\hline Resistant & $22(21.8)$ & $23(95.8)$ & $1(100)$ & $46(36.5,28.6-45.2)$ \\
\hline Intermediate & $29(28.7)$ & $1(4.2)$ & 0 & $30(23.8,17.2-31.9)$ \\
\hline \multicolumn{5}{|l|}{ Amphotericin B } \\
\hline Resistant & $89(88.1)$ & $24(100)$ & $1(100)$ & $114(90.5,84-94.5)$ \\
\hline Intermediate & $12(11.8)$ & 0 & 0 & $12(9.5,5.5-15.91)$ \\
\hline \multicolumn{5}{|l|}{ Fluconazole } \\
\hline Susceptible & $63(62.3)$ & 0 & 0 & $63(50,41.4-58.6)$ \\
\hline Resistant & $29(28.7)$ & $24(100)$ & $1(100)$ & $54(42.9,34.5-51.6)$ \\
\hline Intermediate & $9(8.9)$ & 0 & 0 & $9(7.1,3.8-13.02)$ \\
\hline \multicolumn{5}{|l|}{ Nystatin } \\
\hline Susceptible & $43(42.6)$ & $4(16.7)$ & 0 & $47(37.3,29.4-46)$ \\
\hline Resistant & $4(3.9)$ & $2(8.3)$ & 0 & $6(4.8,2.2-10)$ \\
\hline Intermediate & $54(53.5)$ & $18(75)$ & $1(100)$ & $73(57.9,49.2-66.2)$ \\
\hline \multicolumn{5}{|l|}{ Clotrimazole } \\
\hline Susceptible & $61(60.4)$ & $4(16.7)$ & 0 & $65(51.6,42.9-60.14)$ \\
\hline Resistant & $15(14.9)$ & $18(75)$ & 0 & $33(26.2,19.3-34.5)$ \\
\hline Intermediate & $25(24.8)$ & $2(8.3)$ & $1(100)$ & $28(22.2,15.85-30.2)$ \\
\hline
\end{tabular}

\section{Discussion}

This study revealed that $C$. albicans $(80.6 \%, 101 / 126)$, C. glabrata $(19.05 \%, 24 / 126)$, and $C$. krusei $(0.79 \%$, $1 / 126)$ were prevalent among pregnant women that had clinically confirmed vulvovaginitis, especially 
those in the third trimester $(49.2 \%, 62 / 126)$.

All Candida isolated in this study were resistant to amphotericin B, and all non-albicans Candida were resistant to itraconazole, amphotericin $B$, and fluconazole.

The use of a chromogenic media has enabled the isolation to species level of clinically relevant Candida species in this setting and presents options for its adoption for routine clinical use. In addition to commonly reported $C$. albicans, this study has reported presence of multidrug resistant non-albicans Candida - resistant even to the commonly used antifungals. Pregnant women in the third trimester were mostly affected by VVC unlike a similar study in Peshawar which reported most infections in the second trimester.[26]

C. glabrata is intrinsically of intermediate resistance to fluconazole as a result of the induction of efflux pumps on exposure to azoles which are only fungistatic.[27] Globally, there has been a surge in MDR $C$. glabrata associated with prior fluconazole exposure. [28] In the African context were the cheaper azole antifungals are frequently utilized, resistance to multiple antifungals would be expected. Similarly, $C$. krusei are intrinsically resistant to fluconazole, [29] and their emergence is a sign of clinical failure.

Treatment options for MDR Candida infections especially among pregnant women are limited with expert recommendations lacking in evidence.[30] Expert guidelines have few evidence-based data to guide their recommendations, especially for systemic infections.[30] MDR C. glabrata were isolated from vaginal discharge of pregnant women in this study setting. Colonization of the vagina with such strains has been associated with increased risk of morbidity and mortality in the infants. Such infections have been shown to have an elevated clinical failure rate when they cause systemic infections. [31] In such cases, it's recommended that liposomal amphotericin $B$ is used in addition to managing the source of the infection. [32] However, in this study, they are also resistant to amphotericin B - a finding similar to that of an earlier study.[21]

There was a high number of isolates showing an intermediate level of resistance to each of the antifungal drugs tested. This is an indicator of emergence of high rates of resistance to antifungal drugs. [33]

\section{Conclusion}

Given the emergence of drug resistant non Candida albicans in the causation of VVC in this setting, there is need to change treatment approaches used in the management of VVC especially among pregnant women in the third trimester in this region. Effective therapeutic measures should be put in place to prevent colonization of the newborn with MDR Candida strains. Further research is needed to fully understand the mechanisms of resistance among these strains, and their distribution in the population served by this hospital. 


\section{Abbreviations}

C. albicans - Candida albicans

C. glabrata - Candida glabrata

C. tropicalis - Candida tropicalis

C. krusei - Candida krusei

VVC - Vulvovaginal candidiasis

MDR - Multidrug resistant

NSEQA - National System for External Quality Assessment

CLSI - Clinical Laboratory Standards Institute

C. tropicalis - Candida tropicalis

C. krusei - Candida krusei

VVC - Vulvovaginal candidiasis

MDR - Multidrug resistant

NSEQA - National System for External Quality Assessment

CLSI - Clinical Laboratory Standards Institute

\section{Declarations}

\section{Acknowledgements}

We acknowledge the clinical microbiology laboratory (Busitema University) for the laboratory experiments. I'm also indebted to the staff of antenatal clinic Mbale Regional Referral Hospital, study participants for providing the specimen and the Department of Microbiology and Immunology Busitema University.

\section{Funding}

This study was funded by Busitema University and Makerere University in Uganda. The funding bodies had no role in the design of the study, collection, analysis, interpretation of data, and in writing of the manuscript. 


\section{Availability of data and materials}

All data generated or analysed during this study are included in this published article. The datasets used and/or analyzed during the current study are available from the corresponding author on reasonable request.

\section{Authors' contributions}

WJ conducted laboratory testing and participated in the writing of the manuscript. IJ and JBK supervised the laboratory testing and participated in the writing of the manuscript. JBK proofread and formatted the manuscript for submission. All authors have read and approved the manuscript.

\section{Ethics approval and consent to participate}

Written consent was obtained from all study participants or their guardians (for those under the age of 18 years). Ethics approval to conduct this study was obtained from Higher Research and Ethics Committee of the University of Cape Town, Faculty of Health Sciences (HREC 014/2015).

\section{Competing interests}

The authors declare that they have no competing interests.

\section{References}

1. Zisova LG, Chokoeva AA, Amaliev GI, Petleshkova PV, Miteva-Katrandzhieva Tcapital Em C, Krasteva MB, Uchikova EH, Kouzmanov AH, Ivanova ZV: Vulvovaginal Candidiasis in Pregnant Women and its Importance for Candida Colonization of Newborns. Folia Med (Plovdiv) 2016, 58(2):108-114.

2. Beigi RH, Meyn LA, Moore DM, Krohn MA, Hillier SL: Vaginal yeast colonization in nonpregnant women: a longitudinal study. Obstetrics and gynecology 2004, 104(5 Pt 1):926-930.

3. Parveen N, Munir AA, Din I, Majeed R: Frequency of vaginal candidiasis in pregnant women attending routine antenatal clinic. Journal of the College of Physicians and Surgeons--Pakistan : JCPSP 2008, 18(3):154-157.

4. Nikolov A, Shopova E, Museva A, Dimitrov A: [Vaginal candida infection in the third trimester of pregnancy]. Akusherstvo i ginekologiia 2006, 45(6):7-9.

5. de Leon EM, Jacober SJ, Sobel JD, Foxman B: Prevalence and risk factors for vaginal Candida colonization in women with type 1 and type 2 diabetes. BMC infectious diseases 2002, 2:1. 
6. Bitew A, Abebaw Y: Vulvovaginal candidiasis: species distribution of Candida and their antifungal susceptibility pattern. BMC Womens Health 2018, 18(1):94.

7. Consolaro ME, Albertoni TA, Svidzinski AE, Peralta RM, Svidzinski TI: Vulvovaginal candidiasis is associated with the production of germ tubes by Candida albicans. Mycopathologia 2005, 159(4):501507.

8. Tang $Y, Y u F$, Huang L, Hu Z: The changes of antifungal susceptibilities caused by the phenotypic switching of Candida species in 229 patients with vulvovaginal candidiasis. Journal of Clinical Laboratory Analysis 2019, 33(1):e22644.

9. Ge SH, Wan Z, Li J, Xu J, Li RY, Bai FY: Correlation between azole susceptibilities, genotypes, and ERG11 mutations in Candida albicans isolates associated with vulvovaginal candidiasis in China. Antimicrob Agents Chemother 2010, 54(8):3126-3131.

10. Whaley SG, Berkow EL, Rybak JM, Nishimoto AT, Barker KS, Rogers PD: Azole Antifungal Resistance in Candida albicans and Emerging Non-albicans Candida Species. Frontiers in microbiology 2017, 7:2173-2173.

11. Chokoeva A, Kouzmanov A, Ivanova Z, Zisova L, Amalie G, Petleshkova P, Miteva-Katrandzhieva T, Krasteva M, Uchikova E: [Investigation on Antifungal Susceptibility of Candida Yeasts in Pregnant Patients with Confirmed Vulvovaginal Candidiasis and Their Newborns.]. Akush Ginekol (Sofiia) 2016, 55(4):20-29.

12. Amouri I, Sellami H, Borji N, Abbes S, Sellami A, Cheikhrouhou F, Maazoun L, Khaled S, Khrouf S, Boujelben $Y$ et al: Epidemiological survey of vulvovaginal candidosis in Sfax, Tunisia. Mycoses 2011, 54(5):e499-e505.

13. Pfaller MA, Jones RN, Castanheira M: Regional data analysis of Candida non-albicans strains collected in United States medical sites over a 6-year period, 2006-2011. Mycoses 2014, 57(10):602-611.

14. Pfaller MA, Diekema DJ, Gibbs DL, Newell VA, Nagy E, Dobiasova S, Rinaldi M, Barton R, Veselov A: Candida krusei, a multidrug-resistant opportunistic fungal pathogen: geographic and temporal trends from the ARTEMIS DISK Antifungal Surveillance Program, 2001 to 2005. Journal of clinical microbiology 2008, 46(2):515-521.

15. Guzel AB, Aydin M, Meral M, Kalkanci A, llkit M: Clinical characteristics of Turkish women with Candida krusei vaginitis and antifungal susceptibility of the C. krusei isolates. Infect Dis Obstet Gynecol 2013, 2013:698736.

16. lavazzo C, Gkegkes ID, Zarkada IM, Falagas ME: Boric Acid for Recurrent Vulvovaginal Candidiasis: The Clinical Evidence. Journal of Women's Health 2011, 20(8):1245-1255. 
17. Vroumsia T, Djaouda M, Gaké B, Ebang Menye D, Akoachere Christie E, Tambe E, Vughosu Akindoh V, Njiné T: Prevalence of Vulvovaginal Candidiasis amongst pregnant women in Maroua (Cameroon) and the sensitivity of Candida albicans to extracts of six locally used antifungal plants; 2013.

18. Falagas ME, Betsi GI, Athanasiou S: Probiotics for prevention of recurrent vulvovaginal candidiasis: a review. The Journal of antimicrobial chemotherapy 2006, 58(2):266-272.

19. Nelson M WW, Margaret MW.: Prevalence of Vaginal Candidiasis and Determination of the Occurrence of Candida Species in Pregnant Women Attending the Antenatal Clinic of Thika District Hospital, Kenya. Open J Med Microbiol 2013:264-272.

20. Yesudhason BL, Mohanram K: Candida tropicalis as a Predominant Isolate from Clinical Specimens and its Antifungal Susceptibility Pattern in a Tertiary Care Hospital in Southern India. Journal of clinical and diagnostic research : JCDR 2015, 9(7):DC14-DC16.

21. Mukasa KJ, Herbert I, Daniel A, Sserunkuma KL, Joel B, Frederick B: Antifungal Susceptibility Patterns of Vulvovaginal Candida species among Women Attending Antenatal Clinic at Mbarara Regional Referral Hospital, South Western Uganda. British microbiology research journal 2015, 5(4):322-331.

22. Devi L, Maheshwari M: Speciation of Candida species isolated from clinical specimens by using Chrom agar and conventional methods, vol. 4; 2018.

23. Hu Z, Zhou W, Mu L, Kuang L, Su M, Jiang Y: Identification of cytolytic vaginosis versus vulvovaginal candidiasis. Journal of lower genital tract disease 2015, 19(2):152-155.

24. Vijaya D, Dhanalakshmi TA, Kulkarni S: Changing trends of vulvovaginal candidiasis. J Lab Physicians 2014, 6(1):28-30.

25. Morris AJ, Rogers K, McKinney WP, Roberts SA, Freeman JT: Antifungal susceptibility testing results of New Zealand yeast isolates, 2001-2015: Impact of recent CLSI breakpoints and epidemiological cut-off values for Candida and other yeast species. J Glob Antimicrob Resist 2018, 14:72-77.

26. Khan M, Ahmed J, Gul A, Ikram A, Lalani FK: Antifungal susceptibility testing of vulvovaginal Candida species among women attending antenatal clinic in tertiary care hospitals of Peshawar. Infect Drug Resist 2018, 11:447-456.

27. Tumbarello M, Sanguinetti M, Trecarichi EM, La Sorda M, Rossi M, de Carolis E, de Gaetano Donati K, Fadda G, Cauda R, Posteraro B: Fungaemia caused by Candida glabrata with reduced susceptibility to fluconazole due to altered gene expression: risk factors, antifungal treatment and outcome. The Journal of antimicrobial chemotherapy 2008, 62(6):1379-1385.

28. Kullberg BJ, Arendrup MC: Invasive Candidiasis. The New England journal of medicine 2015, 373(15):1445-1456. 
29. Arendrup MC, Patterson TF: Multidrug-Resistant Candida: Epidemiology, Molecular Mechanisms, and Treatment. The Journal of Infectious Diseases 2017, 216(suppl_3):S445-S451.

30. Pappas PG, Kauffman CA, Andes DR, Clancy CJ, Marr KA, Ostrosky-Zeichner L, Reboli AC, Schuster MG, Vazquez JA, Walsh TJ et al: Executive Summary: Clinical Practice Guideline for the Management of Candidiasis: 2016 Update by the Infectious Diseases Society of America. Clinical infectious diseases : an official publication of the Infectious Diseases Society of America 2016, 62(4):409-417.

31. Lewis JS, 2nd, Wiederhold NP, Wickes BL, Patterson TF, Jorgensen JH: Rapid emergence of echinocandin resistance in Candida glabrata resulting in clinical and microbiologic failure. Antimicrobial agents and chemotherapy 2013, 57(9):4559-4561.

32. Alexander BD, Johnson MD, Pfeiffer CD, Jimenez-Ortigosa C, Catania J, Booker R, Castanheira M, Messer SA, Perlin DS, Pfaller MA: Increasing echinocandin resistance in Candida glabrata: clinical failure correlates with presence of FKS mutations and elevated minimum inhibitory concentrations. Clinical infectious diseases : an official publication of the Infectious Diseases Society of America 2013, 56(12):1724-1732.

33. Whaley SG, Berkow EL, Rybak JM, Nishimoto AT, Barker KS, Rogers PD: Azole Antifungal Resistance in Candida albicans and Emerging Non-albicans Candida Species. Frontiers in microbiology 2016, 7:2173. 